\title{
Estudio a 2 años para medir la eficacia del fentanilo intranasal en pectina en el dolor irruptivo en enfermos con dolor no oncológico de edad avanzada
}

\author{
R. Salazar Vecino \\ Servicio de Anestesiología, Reanimación y Clínica de Dolor. Hospital Comarcal de Inca. Islas Baleares
}

Salazar Vecino R. Estudio a 2 años para medir la eficacia del fentanilo intranasal en pectina en el dolor irruptivo en enfermos con dolor no oncológico de edad avanzada. Rev Soc Esp Dolor 2015; 22(3): 106-111.

\begin{abstract}
Patients with moderate-to-severe pain in non-cancer conditions usually receive opioids for pain control. Many of these patients also suffer breakthrough pain, with the same characteristics as cancer pain. Their breakthrough pain is mainly related to wandering and walking, which in this study we treated with fentanyl pectin nasal spray.

The study initially included 22 elderly patients $(20$ men and 2 women) with non chronic cancer pain with a mean age of 74.7 (60-88) years in a 24 month follow-up.

Lack of problems of abuse of these drugs was confirmed probably by the proper selection of patients and health education that enabled them to distinguish exacerbations of their baseline pain from the breakthrough pain episodes and its specific treatment.

Fentanyl pectin nasal spray showed good tolerability, and improved in more than 4 points relief of breakthrough pain due to wandering and walking, also improved quality of life and sleep in our 24 months study in non-cancer patients.
\end{abstract}

Key words: Non-cancer breakthrough pain. Opioids for analgesia of pain at rest. Fast acting fentanyl.

\section{RESUMEN}

Los enfermos con dolor de moderado a intenso en patologías no oncológicas reciben habitualmente opioides para el control de su dolor. Muchos de ellos también padecen dolor irruptivo de las mismas características que el oncológico, sobre todo incidental a la deambulación, que en este estudio tratamos con fentanilo nasal en pectina.

Se trata de 22 enfermos en la visita basal -20 mujeres (M) y 2 varones $(\mathrm{V})$ - con dolor crónico no oncológico, de edad avanzada 74,7 (60-88), en un seguimiento de 24 meses.

Constatamos la ausencia de problemas de abusos probablemente por la adecuada selección de los pacientes y la educación sanitaria que les permitió distinguir las reagudizaciones del dolor de base, del dolor irruptivo y su tratamiento específico.

El fentanilo nasal en pectina demostró buena tolerancia y mejoró en más de 4 puntos el alivio del dolor irruptivo a la deambulación, la calidad de vida y del sueño en nuestro seguimiento a 24 meses en pacientes no oncológicos.

Palabras clave: Dolor irruptivo no oncológico. Opioides para la analgesia del dolor en reposo. Fentanilos de liberación inmediata.

\section{INTRODUCCIÓN}

En el tratamiento del dolor de moderado a intenso, tanto en el causado por un proceso oncológico como en los enfermos no oncológicos, está indicado el tratamiento con opioides mayores, puesto que su utilización depende de la intensidad del dolor y no del pronóstico (1). Este supuesto, ampliamente aceptado por la comunidad científica, se da también cuando hablamos de dolor irruptivo oncológico (DIO), pero ya no es tan firme cuando se trata de este mismo dolor en enfermos no oncológicos (2). Y eso a pesar de que las características del dolor no oncológico son similares a las del dolor irruptivo oncológico (3): 
- Una prevalencia parecida de entre el 60 y el $75 \%$.

- Mismo número de episodios, como muestra Davies en la última revisión de 2013 (4), en este caso van de 1 episodio al mes, hasta 5 o 6 al día (media de 2,6 al día).

- Una duración de 2-3 minutos a 2 horas.

- Las mismas características de dolor somático, neuropático o mixto, siendo más frecuente el de tipo incidental, en relación con una actividad motora, y por ello más predecible, pues el grueso de los pacientes lo son por afectación músculo-esquelética.

En las Unidades de Dolor hay una gran variedad de patologías en las que tenemos controlado el dolor de base, entre otros medicamentos, con opioides mayores: neuralgias del trigémino, cefaleas en racimos, síndrome del dolor regional complejo (SDRC), rehabilitación tras la colocación de una prótesis, pero sobre todo lumbalgias severas ya por síndromes post-laminectomía, artrosis generalizadas o hernias discales, cervicobraquialgias, coxalgias y gonalgias pendientes de prótesis o inoperables, en las que el dolor irruptivo a la deambulación o la realización de actividades sencillas de la vida, supone un problema y un reto, dada la merma en la calidad de vida que supone para nuestros pacientes.

En estos pacientes los fentanilos de acción rápida y en concreto el fentanilo intranasal en pectina, por su fácil aplicación, liberación inmediata y rápido inicio de acción, al adaptarse más al perfil del dolor irruptivo, puede aportar un importante alivio del dolor incidental, aumentando con ello la calidad de vida de nuestros pacientes al mejorar la deambulación, la posibilidad de realizar tareas habituales y domésticas, y la calidad de sueño $(5,6)$.

\section{MATERIAL Y MÉTODOS}

Se trata de un estudio que en principio era de 6 semanas y que posteriormente se alargó a 12, 18 y 24 meses (Tabla I),

TABLA I. DIAGNÓSTICOS Y MEDICACIÓN

\begin{tabular}{|c|c|c|c|c|c|}
\hline Tiempo & 0 semanas & 6 semanas & 12 meses & 18 meses & 24 meses \\
\hline N. ${ }^{\circ}$ pacientes & $22(20 \mathrm{M} / 2 \mathrm{~V})$ & $20(18 \mathrm{M} / 2 \mathrm{~V})$ & $13(11 \mathrm{M} / 2 \mathrm{~V})$ & $11(9 \mathrm{M} / 2 \mathrm{~V})$ & $10(8 \mathrm{M} / 2 \mathrm{~V})$ \\
\hline Edad & $60-88(74,7)$ & $60-88(74,2)$ & $61-89(75,7)$ & $62-87(75,9)$ & $62-88(77,3)$ \\
\hline Diagnóstico & \multicolumn{5}{|c|}{ Número de pacientes } \\
\hline Aplast. vertebral & 9 & 8 & 3 & 4 & 4 \\
\hline Estenosis de canal & 8 & 7 & 4 & 4 & 3 \\
\hline Gonalgias/Coxalgias & 6 & 6 & 4 & 3 & 2 \\
\hline Espondilolistesis & 6 & 4 & 4 & 3 & 2 \\
\hline Hernia discal & 5 & 4 & 2 & 1 & 1 \\
\hline Secuelas post-artrodesis & 3 & 3 & 3 & 2 & 2 \\
\hline Rehabilitación PTR & 1 & 1 & 2 & 0 & 1 \\
\hline Opioide de base & \multicolumn{5}{|c|}{ Número de pacientes } \\
\hline Oxicodona/Naloxona & $\begin{array}{c}15 \\
5 / 2,5-60 / 30 \mathrm{mg} / \mathrm{dí} a\end{array}$ & $\begin{array}{c}14 \\
5 / 2,5-60 / 30 \mathrm{mg} / \mathrm{día}\end{array}$ & $\begin{array}{c}8 \\
5 / 2,5-40 / 20 \mathrm{mg} / \mathrm{día}\end{array}$ & $\begin{array}{c}8 \\
5 / 2,5-40 / 20 \mathrm{mg} / \text { día }\end{array}$ & $\begin{array}{c}7 \\
5 / 2,5-40 / 20 \mathrm{mg} / \mathrm{dí}\end{array}$ \\
\hline Tapentadol & $\begin{array}{c}3 \\
100-200 \mathrm{mg} / \mathrm{día}\end{array}$ & $\begin{array}{c}2 \\
100-200 \text { mg/día }\end{array}$ & $\begin{array}{c}1 \\
200 \mathrm{mg} / \mathrm{día}\end{array}$ & 0 & 0 \\
\hline Hidromorfona & $\begin{array}{c}2 \\
8-16 \mathrm{mg} / \mathrm{día}\end{array}$ & $\begin{array}{c}2 \\
8-16 \mathrm{mg} / \mathrm{día}\end{array}$ & $\begin{array}{c}3 \\
8-16 \mathrm{mg} / \mathrm{día}\end{array}$ & $\begin{array}{c}2 \\
4-8 \mathrm{mg} / \mathrm{día}\end{array}$ & $\begin{array}{c}2 \\
4-8 \mathrm{mg} / \mathrm{día}\end{array}$ \\
\hline Fentanilo TTS & $\begin{array}{c}2 \\
25-75 \mu \mathrm{g}\end{array}$ & $\begin{array}{c}2 \\
25-75 \mu g\end{array}$ & $\begin{array}{c}1 \\
25 \mu \mathrm{g}\end{array}$ & $\begin{array}{c}1 \\
25 \mu \mathrm{g}\end{array}$ & $\begin{array}{c}1 \\
12 \mu \mathrm{g}\end{array}$ \\
\hline \multicolumn{6}{|c|}{ Tratamiento del dolor irruptivo con fentanilo nasal en pectina } \\
\hline $\begin{array}{l}\text { Dosis media por episodio } \\
\text { Dosis utilizadas }\end{array}$ & & $\begin{array}{c}120 \mu \mathrm{g} \\
100-200 \mu \mathrm{g}\end{array}$ & $\begin{array}{c}190 \mu \mathrm{g} \\
100-400 \mu \mathrm{g}\end{array}$ & $\begin{array}{c}220 \mu \mathrm{g} \\
100-400 \mu \mathrm{g}\end{array}$ & $\begin{array}{c}210 \mu \mathrm{g} \\
100-400 \mu \mathrm{g}\end{array}$ \\
\hline $\begin{array}{l}\text { Media de aplicaciones } \\
\text { Frecuencia de la dosis }\end{array}$ & & $\begin{array}{c}\text { 2,6 día } \\
\text { (1 a la sem./4 al día) }\end{array}$ & $\begin{array}{c}\text { 2,4 día } \\
\text { (1 a la sem./4 al día) }\end{array}$ & $\begin{array}{c}2,8 \text { día } \\
\text { (1 a la sem./4 al día) }\end{array}$ & $\begin{array}{c}\text { 2,6 día } \\
\text { (1 al mes/4 al día) }\end{array}$ \\
\hline
\end{tabular}

PTR: prótesis total de rodilla. M: mujer. V: varón. 
para valorar la eficacia del fentanilo nasal en pectina, en el tratamiento del dolor irruptivo a la deambulación de enfermos con distintas patologías no excluyentes, pudiendo padecer 2 o más de ellas (Tabla I), de edad avanzada, con el dolor en reposo controlado con distintos opioides (Tabla I), antidepresivos, anticomiciales, ansiolíticos, AINE, distintos bloqueos con anestésicos locales + corticoides (epidurales lumbares o caudales, infiltraciones sacroiliacas, infiltraciones en rodilla y/o cadera con ácido hialurónico, infiltraciones miofasciales y con toxina botulínica...).

Si bien en las primeras 6 semanas no se modificaron los tratamientos, ni se realizaron técnicas, para asegurar que valorábamos de forma aislada la eficacia específica del fentanilo nasal en pectina, sí que posteriormente se cambiaron y se hicieron infiltraciones, si es que los pacientes lo precisaron, para tener controlado y con el mínimo de efectos secundarios el dolor basal.

Para ello se midió: EVA en reposo y EVA en movimiento a las $0,2,4$ y 6 semanas y a los 12,18 y 24 meses. Se midió a las 0,4 y 6 semanas, 12,18 y 24 meses el cuestionario sobre el estado de salud general SF-12; la escala de calidad de vida EQ-5D; el cuestionario de salud EuroQol-5D; el test de evaluación del grado de incapacidad funcional de Oswestry y el índice de problemas de sueño MOS. Por último, la impresión global del cambio según el paciente (PGIC) a las 6 semanas, 12, 18 y 24 meses.

\section{RESULTADOS}

Todos los parámetros medidos mejoraron clínicamente con significación estadística excepto la EVA en reposo. La EVA en reposo también mejoró clínicamente si bien sin significación estadística. La EVA en reposo pasó de un valor medio al inicio de 1,7 (mínimo 0 - máximo 4); a 1,2 a las 6 semanas; 1,5 a los 12 meses; 1,3 a los 18 meses; y 1,2 a los 2 años (mínimo 0 - máximo 3) (Fig. 1).

La EVA en movimiento tuvo una mejora clínica relevante que además fue estadísticamente significativa

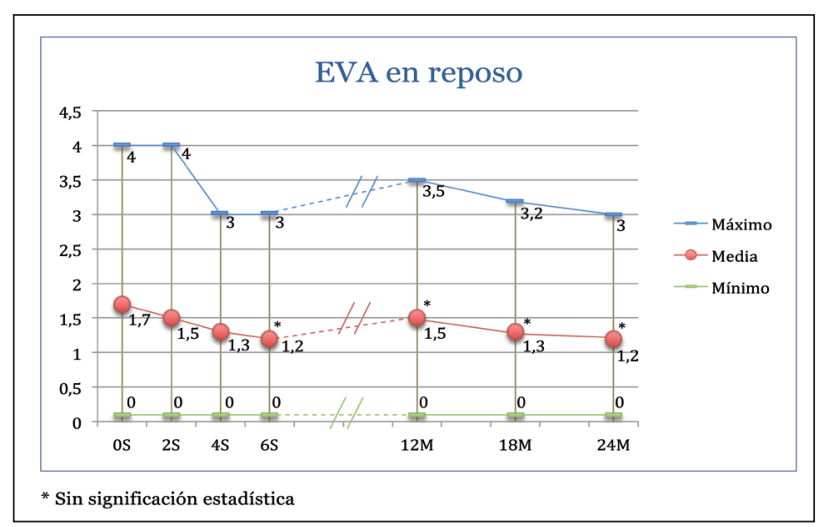

Fig. 1. EVA en reposo. $(\mathrm{p}<0,001)$. Pasó de un valor medio en la visita basal de 6,6 puntos (mínimo 4 - máximo 9); a 2,8 a las 6 semanas; 2,5 a los 12 meses; 2,7 a los 18 meses; y 2,4 a los 2 años (mínimo 1 - máximo 4,8) (Fig. 2).

El cuestionario de salud general SF-12 mejoró de forma clínicamente relevante; dicha mejora fue estadísticamente significativa $(\mathrm{p}<0,001)$. Mejoró de un valor medio en la visita basal de 33,33 (mínimo 8,3 - máximo 60); a 46,2 a las 6 semanas; 47,4 a los 12 meses; 46,8 a los 18 meses; y 47,1 a los 2 años (mínimo 8,3 - máximo 79,5) (Fig. 3).

El cuestionario de calidad de vida EQ-5D mejoró de 0,4 en la visita basal (mínimo 0,1 - máximo 0,8); a 0,6 a las 6 semanas y se mantuvo la mejora al 0,6 en todas las mediciones posteriores hasta los 2 años. Dicha mejora también tuvo significación estadística ( $<<0,001)$ (Fig. 4).

El estado de salud del EuroQol-5D aumentó con significación estadística $(\mathrm{p}<0,001)$ de 40,7 en la visita basal (mínimo 20 - máximo 70); a 50,6 a las 6 semanas; 51,3 a los 12 meses; a 50,8 a los 18 meses; y 50,9 a los 2 años (mínimo 22 - máximo 77) (Fig. 5).

En el test de evaluación funcional de Oswestry se ve cómo el grado de incapacidad desciende de una media en la visita basal de 56,5 (mínimo 30 - máximo 80); a 47,4 a

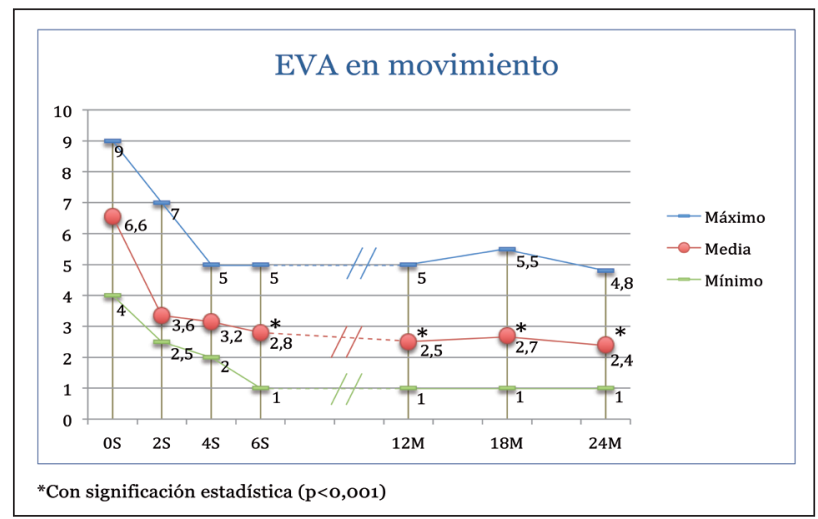

Fig. 2. EVA en movimiento.

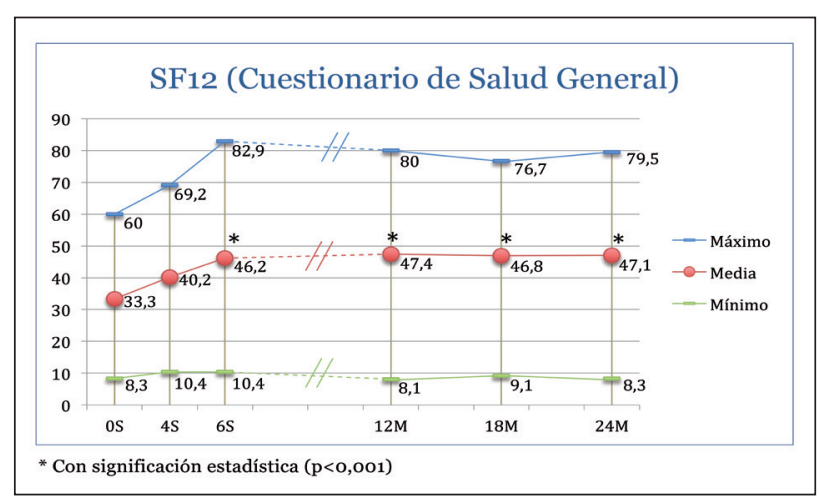

Fig. 3. SF12 (Cuestionario de Salud General). 


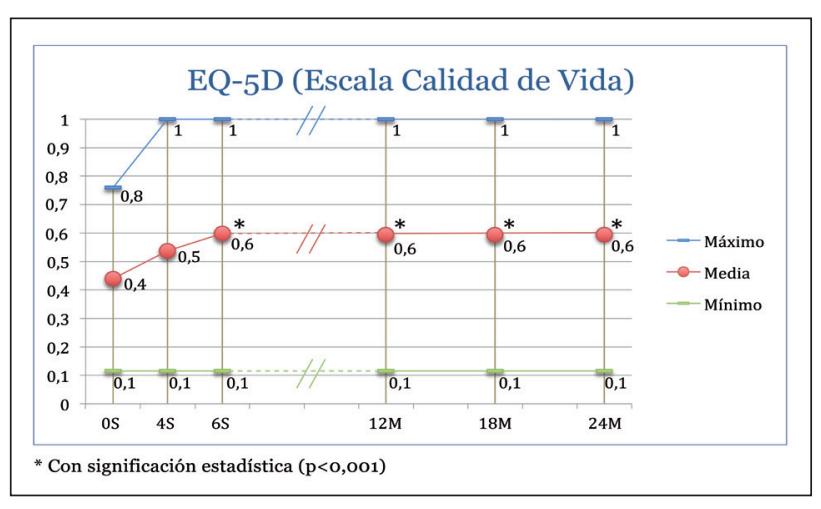

Fig. 4. EQ-5D (Escala Calidad de Vida).

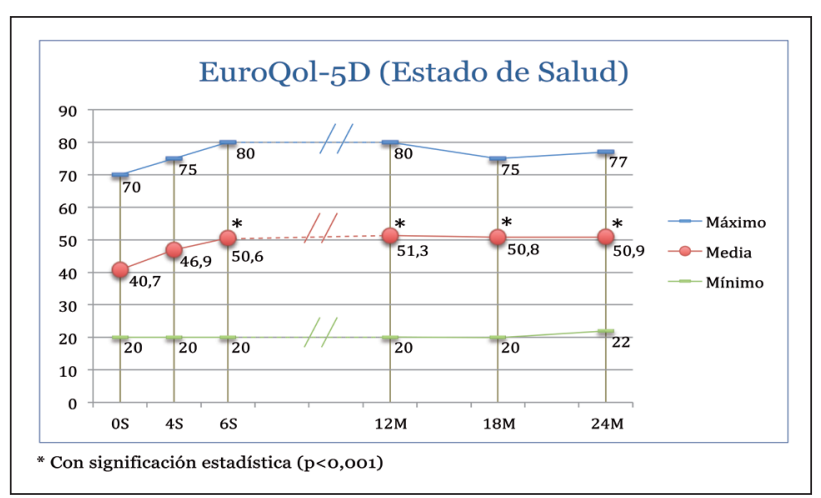

Fig. 5. EuroQol-5D (estado de salud).

las 6 semanas; 47,8 a los 12 meses; 47,9 a los 18 meses; y 47,8 a los 2 años (mínimo 23 - máximo 77). Dicha mejora tuvo significación estadística ( $\mathrm{p}<0,005)$ (Fig. 6).

Las incidencias en el sueño (MOS), también mejoraron con significación estadística ( $\mathrm{p}<0,001)$ de 33,6 en la visita basal (mínimo 9,4 - máximo 75,5); a 24,6 a las 6 semanas; 24,4 al año; 24,4 al año y medio; y 24,5 a los 2 años (mínimo 5 - máximo 55) (Fig. 7).

Por último en el PGIC o grado de satisfacción del paciente (que se evaluó a las 6 semanas, año, año y medio y 2 años) se confirmó que prácticamente la totalidad de los pacientes se encontraban mejor o mucho mejor con el tratamiento (Fig. 8).

\section{DISCUSIÓN}

El diseño del estudio fue en principio de 6 semanas, pero con posterioridad y para valorar la tolerancia y si había algún problema de adicción, dados los pocos estudios existentes de utilización de fentanilos de liberación inmediata a largo plazo, se decidió alargarlo a un año, posteriormente a un año y medio y a dos, teniendo intención de continuar el seguimiento en el futuro. Esto obligó a poder cambiar

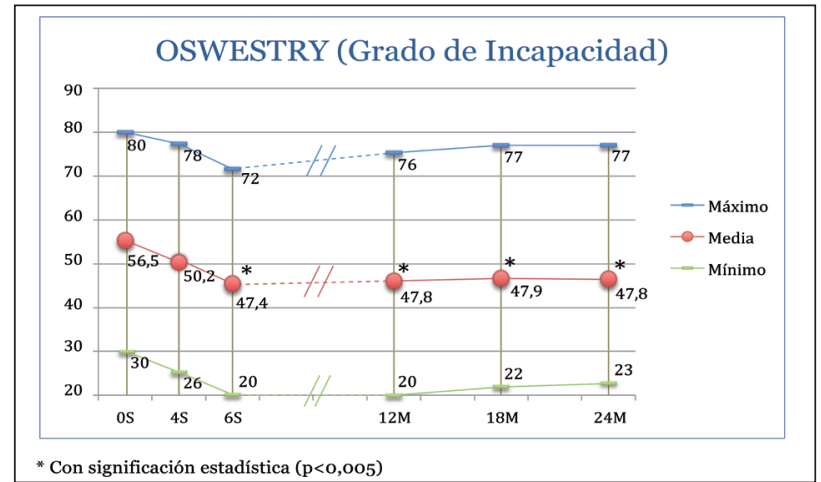

Fig. 6. Oswestry (grado de incapacidad).

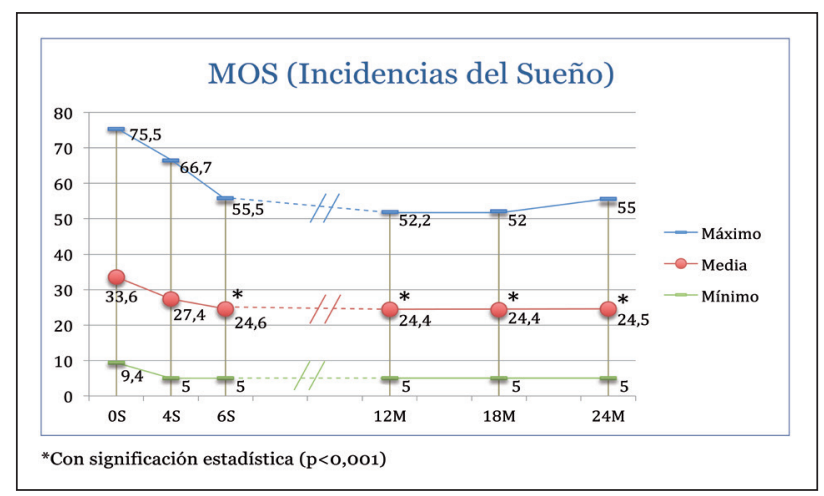

Fig. 7. MOS (incidencias del sueño).

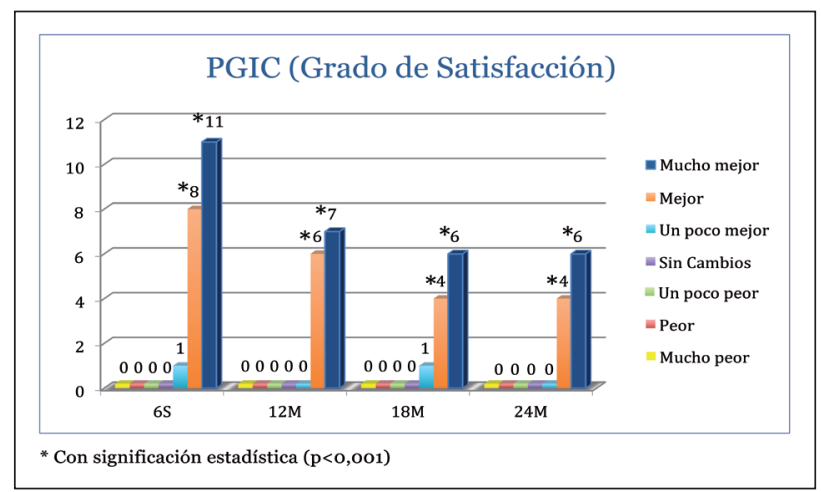

Fig. 8. PGIC (grado de satisfacción).

los tratamientos de los pacientes en función de la evolución del dolor. Si tenían una reagudización se les modificaba la pauta analgésica o, si era posible, se les hacía una infiltración y, al revés, si constatábamos una importante mejoría se les rebajaba la medicación. Pero en todo momento se mantuvo la posibilidad de utilizar el fentanilo nasal en pectina para el dolor irruptivo que, por supuesto, seguían teniéndolo y distinguían muy bien de las reagudizaciones del dolor basal. 
De este modo, pasadas 6 semanas para mantener el dolor de base controlado, si el paciente aquejaba un dolor localizado, por ejemplo en la cadera y no iba a ser operado en los próximos meses, se le hacía una infiltración de la misma con ácido hialurónico de larga duración hasta cada 6 meses, o una infiltración caudal o lumbar si aumentaba la radiculopatía (una tanda de 3 al año) o infiltraciones con toxina botulínica en las contracturas del cuadrado de los lomos o del psoas iliaco en las secuelas post-artrodesis. Con ello se mantenía el dolor en reposo controlado e incluso, como se ha dicho, se pudo bajar la medicación, que también se hacía, si el paciente manifestaba cualquier efecto secundario (somnolencia), para mantener en todo momento el máximo de funcionalidad.

La causa de abandono de la utilización del fentanilo de liberación inmediata, en las 6 primeras semanas, 2 casos, fue por efectos secundarios (náuseas y mareos).

En el transcurso del primer año, tras esas 6 semanas abandonaron 7 pacientes la utilización del fentanilo de liberación inmediata:

- 4 por desaparición del dolor:

- 2 de las gonalgias por operarse de prótesis total de rodilla (PTR).

- 1 de los aplastamientos vertebrales, por desaparición del dolor.

- 1 de las hernias tras infiltración transforaminal.

- 1 por falta de eficacia: estenosis de canal con gran claudicación, que se operó poco después.

- 1 por problemas económicos.

- 1 por éxitus.

En el periodo que va del año al año y medio, abandonaron el tratamiento 2 pacientes, muy frágiles. En el de más edad, 89 años, dada la limitada vida que podía llevar, se aconsejó la retirada de gran parte de la medicación que tomaba.

Entre los 18 y los 24 meses hubo otro fallecimiento, por lo que a los 2 años han llegado 10 pacientes.

Llama la atención que fuera de los brotes de reagudización las cifras en la valoración del dolor, una vez estabilizado, prácticamente no cambian. También, cómo en algunos enfermos el control del dolor en reposo se consigue con apenas un comprimido al día de oxicodona/naloxona de $5 / 2,5$, por la mañana, que le cubre las horas en que están activos y luego usan el fentanilo nasal en pectina en una o dos ocasiones para realizar las tareas domésticas y salir a la calle, por llevar una vida autónoma.

Otro dato relevante es que en el transcurso del primer año y medio se produjo un incremento de la dosis media por episodio de $120 \mu \mathrm{g}$ a $220 \mu \mathrm{g}$ (a pesar de que un paciente usaba el fentanilo una media de 1 vez a la semana). Consideramos que la explicación no es que se haya creado tolerancia, sino que han ido quedando los pacientes más crónicos, con más dolor a la deambulación y sin posibilidades quirúrgicas: las secuelas post-artrodesis, las estenosis de canal que afectan desde D-12 a S-1 y no se considera apropiado operar dadas las enfermedades concomitantes que padecen (IRC, EPOC, IC, diabéticos...). Sucede lo mismo con respecto a algunos de los pacientes que padecen coxalgia o gonalgia (de los que se han operado 3 y 1 está ya en lista de espera para su otra rodilla), porque de los 18 a los 24 meses ha descendido el consumo de la medicación de base y el número de aplicaciones en las que se ha utilizado el fentanilo nasal, lo que achacamos a que los pacientes han pasado un "muy buen invierno" con menos episodios de reagudización, o quizá que han realizado menos actividad.

\section{CONCLUSIONES}

En nuestro seguimiento a dos años en enfermos de edad avanzada con dolor no oncológico comprobamos que el fentanilo nasal en pectina:

- Mejoró el dolor irruptivo. El EVA disminuyó más de 4 puntos (6,6 de la visita basal a 2,4 visita a 2 años).

- Mejoró ligeramente el dolor en reposo.

- Mejoró la calidad de vida.

- Disminuyó el grado de incapacidad.

- Mejoró la cantidad y calidad de sueño.

- Consiguió una alta satisfacción por parte del paciente.

En nuestra práctica clínica el fentanilo nasal en pectina mejoró el dolor irruptivo a la deambulación de estos pacientes de edad avanzada, en algunos casos, hasta que pudo ser operado y con ello solucionarse su causa (1 de estenosis de canal, 3 de PTR) y en otros, los más mayores, que son conscientes de su estado de salud, permitiéndoles llevar el tipo de vida que les corresponde por edad, ser autónomos y no necesitar ayuda para su cuidado personal, la realización de pequeñas actividades que den sentido a sus vidas, mejorando su calidad de vida y de sueño, con un alto grado de satisfacción.

Indicar también la ausencia de problemas de abusos probablemente por la adecuada selección de los pacientes y la educación sanitaria que les permitió distinguir las reagudizaciones del dolor de base, del dolor irruptivo y su tratamiento específico.

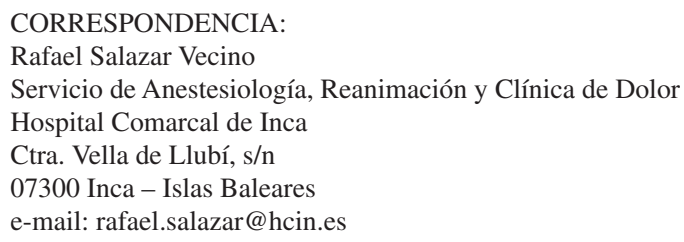




\section{BIBLIOGRAFÍA}

1. Rodríguez López MJ. Manual práctico sobre utilización de opiáceos potentes en el tratamiento del dolor crónico. SED editorial, 2003.

2. Biete i Solà A, Camba Rodríguez M, Escobar Álvarez Y, Gálvez Fuente R, Mañas Rueda A, Rodríguez Sánchez C, et al. Nuevos enfoques en el diagnóstico y tratamiento del dolor irruptivo oncológico: Documento de Consenso. 2012.

3. Cánovas Martínez L, Rodríguez Rodríguez AB, Castro Bande M, Pérez Arviza L, López Soto C, Román Nuñez R.
Tratamiento del dolor irruptivo. Rev Soc Esp Dolor 2012; 19(6):318-24.

4. Davies et al. Brakthrough cancer pain: An observational study of 1000 European oncology patients. Journal of Pain and Sympton Management 2013.

5. Salazar Vecino R. Titulación de opiáceos en dolor crónico no oncológico. VIII curso básico de formación en dolor. Fuengirola, 2014.

6. De Andrés Ares J. Dolor de comienzo súbito: dolor irruptivo. VIII curso básico de formación en dolor. Fuengirola; 2014. 